\title{
A New Cokriging Method for Variable-Fidelity Surrogate Modeling of Aerodynamic Data
}

\author{
Zhong-Hua Han ${ }^{1}$, Ralf Zimmermann ${ }^{2}$ and Stefan Görtz ${ }^{3}$ \\ DLR, German Aerospace Center, D-38108, Braunschweig, Germany
}

\begin{abstract}
Cokriging is a statistical interpolation method for the enhanced prediction of a less intensively sampled primary variable of interest with assistance of intensively sampled auxiliary variables. In the geostatistics community it is referred to as two- or multi-variable kriging. In this paper, a new cokriging method is proposed and used for variable-fidelity surrogate modeling of aerodynamic data obtained with an expensive high-fidelity CFD code, assisted by data computed with cheaper lower-fidelity codes or by gradients computed with an adjoint version of the high-fidelity CFD code, or both. A self-contained derivation as well as the numerical implementation of this new cokriging method is presented and the comparison with the autoregressive model of Kennedy and O'Hagan is discussed. The developed cokriging method is validated against an analytical problem and applied to construct global approximation models of the aerodynamic coefficients as well as the drag polar of an RAE 2822 airfoil based on sampled CFD data. The numerical examples show that it is efficient, robust and practical for the surrogate modeling of aerodynamic data based on a set of CFD methods with varying degrees of fidelity and computational expense. It can potentially be applied in the efficient CFD-based aerodynamic analysis and design optimization of aircraft.
\end{abstract}

\section{Introduction}

Surrogate modeling plays an increasingly important role in different areas of aerospace science and engineering, such as aerodynamic shape optimization, aerodynamic data production, structural design, Multidisciplinary Optimization (MDO) and design of aircraft or spacecraft ${ }^{[1][2]}$. Surrogate modeling can be used to greatly improve the design efficiency when high-fidelity but time-consuming numerical models (e.g. Computational Fluid Dynamic (CFD) models and Finite-Element (FE) structural models) are employed. In addition, it can be very helpful in finding global optima, filtering numerical noise, realizing parallel design optimization and integrating simulation codes of different disciplines into a process chain for engineering design. Here the term "surrogate model" has the same meaning as "response surface model", "metamodel", "approximation model", "emulator" etc. Among the different surrogate modeling techniques, such as quadratic polynomial models or radial basis functions, kriging interpolation ${ }^{[3][4]}$ has received growing popularity due to its good capability of predicting multi-dimensional, highly non-linear responses based on sampled data while providing a useful estimation of the uncertainty of the prediction. Despite the popularity of kriging, further improvements of its efficiency and accuracy are still required before it can be widely used for realistic engineering design problems. Taking the aerodynamic data for load production of an aircraft as an example, building an accurate (at least useable) kriging model based on sampled aerodynamic data obtained with an expensive Navier-Stokes (N-S) CFD code is very demanding, due to the fact that large number of simulations are required. This situation may become even worse with an increase of the size of the problem ${ }^{[5][6][7]}$ (increasing number of independent variables and increasing range of these variables).

Cokriging provides an attractive alterative approach to further improve the efficiency of building a surrogate model. It was initially proposed and applied in the geostatistics community ${ }^{[8][9]}$ for the enhanced prediction of less intensively sampled primary variable of interest with assistance of intensively sampled auxiliary variables. As the

\footnotetext{
${ }^{1}$ Research Scientist, Institute of Aerodynamics and Flow Technology, $\mathrm{C}^{2} \mathrm{~A}^{2} \mathrm{~S}^{2} \mathrm{E}$, Lilienthalplatz 7, 38108 Braunschweig, Germany; Zhong-hua.Han@dlr.de.

${ }^{2}$ Research Scientist, Institute of Aerodynamics and Flow Technology, $\mathrm{C}^{2} \mathrm{~A}^{2} \mathrm{~S}^{2} \mathrm{E}$, Lilienthalplatz 7 , 38108 Braunschweig, Germany; Ralf.Zimmermann@dlr.de.

${ }^{3}$ Team Lead, Aero-Loads Prediction Group, Institute of Aerodynamics and Flow Technology, $\mathrm{C}^{2} \mathrm{~A}^{2} \mathrm{~S}^{2} \mathrm{E}$, Lilienthalplatz 7, 38108 Braunschweig, Germany; Stefan.Goertz@dlr.de; AIAA Member.
} 